'Escuela de Postgrado. Facultad de Medicina. Universidad Católica del Maule. Talca, Chile. ${ }^{2}$ Servicio de Medicina, Hospital Regional de Talca, Talca, Chile. ${ }^{3}$ Facultad de Medicina, Universidad Católica del Maule, Talca, Chile.

aResidente de Medicina Interna, Universidad Católica del Maule. bInterno de Medicina, Universidad Católica del Maule.

Los autores declaran no presentar conflictos de interés.

Recibido el 8 de diciembre de 2015, aceptado el 11 de abril de 2016.

Correspondencia a: Alondra Frías

1 Norte 13 Oriente \# 1990, Talca. Módulo Docente UCM. Teléfono: (0712) 413643 afriasoyarzun@gmail.com

\section{Síndrome de Cushing iatrogénico por corticoides inhalados en paciente VIH}

\author{
ALONDRA FRÍAS ${ }^{1, \mathrm{a}}$, ALEX ORTIZ $^{3, \mathrm{~b}}$, MIRIAM SOTO ${ }^{3, \mathrm{~b}}$, \\ FRANCISCO MUÑOZ ${ }^{2,3}$, CAROLINA CHACÓN $^{2,3}$
}

\section{Cushing syndrome in a HIV patient using inhaled steroids. Report of one case}

\begin{abstract}
We report a 41-year-old man with HIV and a chronic obstructive pulmonary disease, treated for seven months with Fluticasone/Salmeterol and antiretroviral therapy (Lamivudine, Tenofovir, Atazanavir and Ritonavir). While using these medications, the patients developed a Cushing syndrome in a period of five months. After performing laboratory and imaging tests, it was concluded that the most probable cause of the syndrome was the interaction of inhaled steroids with Ritonavir. After discontinuing these medications the syndrome reverted in a period of 8 months.
\end{abstract}

(Rev Med Chile 2016; 144: 936-940)

Key words: Cushing syndrome; Fluticasone; HIV; Ritonavir.
4 n Chile los casos de pacientes viviendo con VIH se han incrementado, durante el año 2011 se registró la mayor tasa de notificaciones en el período que comprendió desde 1984 al 2012. El año 2011 se informó una tasa de 9,6 por cada cien mil habitantes. No obstante, la aparición de nuevos antirretrovirales y un programa nacional ha mejorado tanto la calidad como la esperanza de vida ${ }^{1}$.

En este sentido, el aumento de sobrevida ha permitido que esta patología coexista con diversas comorbilidades; destacando enfermedades respiratorias (neumonía bacteriana, 27\%; enfermedad pulmonar obstructiva crónica (EPOC), 20\%; neumonía por $P$. jirovecii, $9 \%$ y asma, $5 \%)^{2}$.

Desde esta perspectiva, es fundamental considerar las interacciones que pueden existir entre fármacos inhalados y antirretrovirales. Los corticoides inhalados (CI) son el pilar de los principales esquemas terapéuticos en esas co-morbilidades ${ }^{2}$; por lo cual, es necesario conocer cómo pueden interactuar con antirretrovirales.

La presentación de SC en pacientes tratados con corticoide inhalado (CI) es infrecuente, se han informado casos de síndrome de Cushing iatrogénico (SCI) en pacientes VIH en otros países $^{3}$. En nuestro centro se estudió un caso que resultó secundario a la interacción entre un CI y un inhibidor de proteasa (IP), que exponemos a continuación.

\section{Caso clínico}

Paciente de 41 años, de sexo masculino, con antecedentes de VIH, EPOC tabáquico y neumonía grave por $P$. jirovecii. Desde el 2012, en tratamiento con: lamivudina ( $150 \mathrm{mg} /$ día), tenofovir (300 mg/día), atazanavir (300 mg/día) y ritonavir (100 mg/día).

Durante los últimos 7 meses se agregó al tratamiento fluticasona/salmeterol $(1.000 \mathrm{ug} / 100 \mathrm{ug}$ al día), respetando dosis.

Consultó en urgencias, por cuadro de 5 meses de evolución de dolor lumbar bilateral, punzante, no irradiado y EVA 8/10. Asociado a astenia, adinamia y aumento progresivo de peso. Se hospitalizó para estudio.

Ingresó con hipertensión arterial (150/96 $\mathrm{mmHg}$ ) y afebril. El examen físico reveló un IMC $38 \mathrm{~kg} / \mathrm{m}^{2}$ (33 kg/m² hacía 10 meses), perímetro abdominal $(210 \mathrm{~cm})$ y fenotipo cushingoideo (Figura 1). 


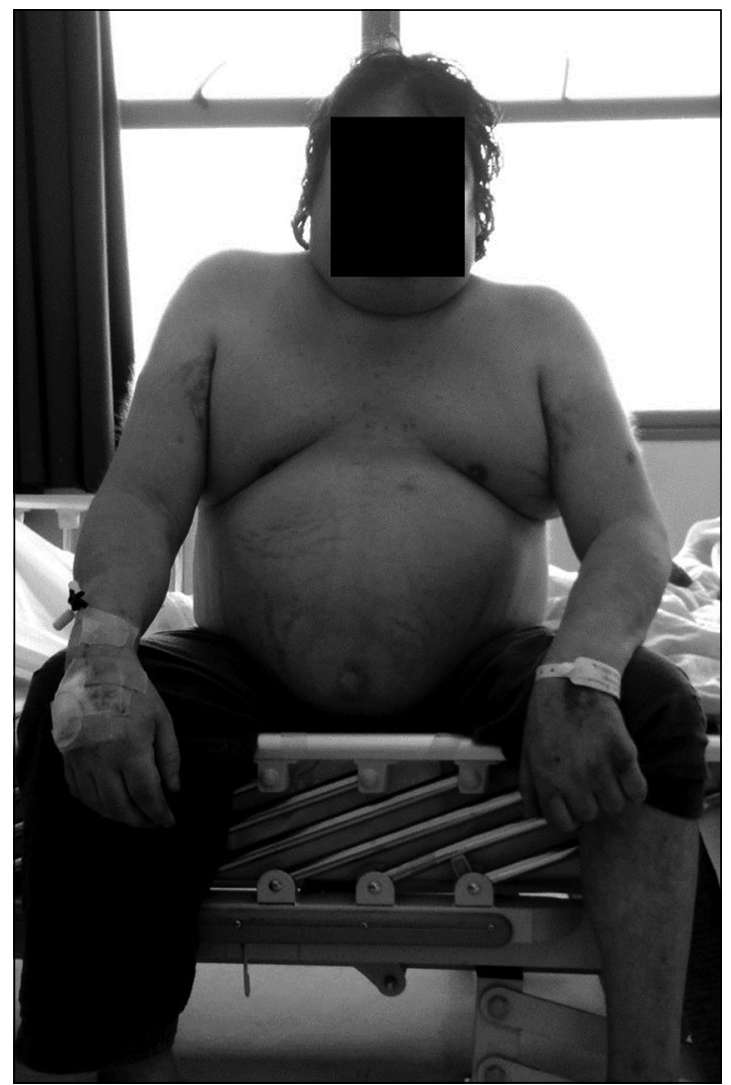

Figura 1. Aspecto al ingreso. Destacan: facie pletórica, jiba dorsal, atrofia muscular de extremidades, estrías violáceas en tórax, abdomen y extremidades.

Se estudió con exámenes de laboratorio, destacando alteración en: cortisol libre urinario de $24 \mathrm{~h}$ (CLU), test de Liddle (TL) en dosis bajas y en ACTH (Tabla 1). Con estos resultados, se realizó una tomografía computada (TC) de abdomen y pelvis con contraste, que evidenció glándulas suprarrenales de forma y tamaño normales.

En radiografías de columna lumbar se pesquisaron fracturas por aplastamiento a nivel de T12 a L3; confirmadas en TC de columna lumbar (Figura 2) y densitometría ósea informó T score: $-4,75$ DS en cuello femoral.

A partir de los hallazgos en el laboratorio endocrinológico e imagenología, se concluyó que el cuadro era secundario a SCI por corticoides exógenos (CE); siendo fluticasona/ritonavir, la asociación más probable.

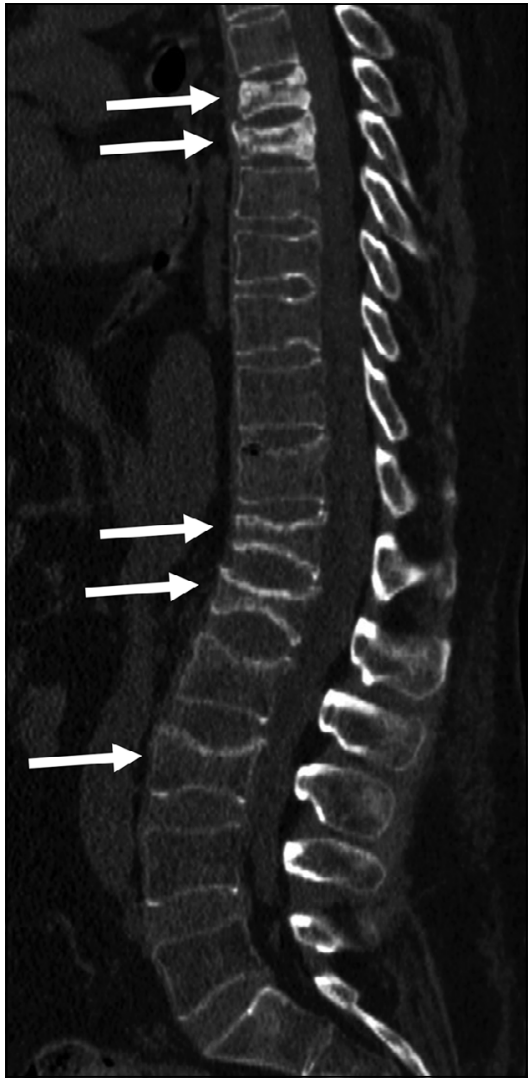

Figura 2. Tomografía computada de columna dorsal y lumbar. Las flechas muestran fracturas por aplastamiento de los cuerpos vertebrales: D5, D6, D12, L1, L3

Por estos motivos, ambos fármacos fueron suspendidos, se suplementó por un mes con cortisol oral (30 mg/día), indacaterol (150 ug/día) reemplazó a fluticasona/salmeterol y se cambió la triterapia a: raltegravir $(800 \mathrm{mg} /$ día $)$, zidovudina/ lamivudina $(600 \mathrm{mg} / 300 \mathrm{mg} /$ día $)$.

Igualmente, recibió calcio/colecalciferol (375 mg/150 UI/día) y ácido ibandrómico (150mg/ mes) por osteoporosis lumbar. Se otorgó el alta al lograr analgesia con buprenorfina 35 ug subcutánea y amitriptilina $25 \mathrm{mg} /$ día.

El eje hipotálamo-hipófisis-adrenal (HHA) fue revisado, a los 3 meses de la suspensión de fluticasona/ritonavir y a los 2 meses sin cortisol; obteniéndose $16 \mathrm{ng} / \mathrm{ml}$ de cortisol basal.

Evolucionó sin dolor, normotenso, con un IMC $35 \mathrm{~kg} / \mathrm{m}^{2}$ y con regresión del fenotipo cushingoideo a los 8 meses de seguimiento. 
Tabla 1

\begin{tabular}{|c|c|c|}
\hline Exámenes & Resultado & Valor de referencia \\
\hline Glicemia ayuno (mg/dl) & 80 & $60-100$ \\
\hline Sodio (mEq/l) & 134 & $136-146$ \\
\hline Potasio (mEq/l) & 4,59 & $3,5-5,1$ \\
\hline Hemoglobina (gr/dl) & 12,4 & $14-18$ \\
\hline Glóbulos blancos (céls/mm³) & 7.400 & $4.000-9.000$ \\
\hline Eosinófilos (\%) & 4 & $4-8$ \\
\hline Proteína C reactiva (mg/dl) & 8,7 & $0-5$ \\
\hline Creatinina (mg/dl) & 0,64 & $0,5-1,2$ \\
\hline GOT (UI/l) & 22 & $10-38$ \\
\hline GPT (UI/I) & 27 & $10-41$ \\
\hline Fosfatasa alcalina (UI/l) & 162 & 40-129 \\
\hline Bilirrubina total (mg/dl) & 0,8 & $0-1,2$ \\
\hline Cortisol libre urinario de 24 h (ug/24 h) & 2,3 & $9-150$ \\
\hline Creatinina en orina de $24 \mathrm{~h}$ ( $\mathrm{mg} / 24 \mathrm{~h}$ ) & 930 & $600-2.000$ \\
\hline ACTH $(p g / m l)$ & 5,0 & $9,0-65,0$ \\
\hline Cortisol basal pre test de Liddle (ng/ml) & 3,8 & $40-230$ \\
\hline Cortisol basal post test de Liddle (ng/ml) & 10,0 & $40-230$ \\
\hline Colesterol total (mg/dl) & 252 & $50-200$ \\
\hline Colesterol HDL (mg/dl) & 57 & $35-55$ \\
\hline Colesterol LDL (mg/dl) & 158 & $0-145$ \\
\hline Triglicéridos (mg/dl) & 187 & $50-200$ \\
\hline Insulinemia ayuno (uUl/ml) & 25,4 & $5-20$ \\
\hline HbA1c (\%) & 4,9 & $4-6$ \\
\hline HOMA IR & 5,0 & $<2,5$ \\
\hline
\end{tabular}

\section{Discusión}

El SC tiene una prevalencia de 39 a 79 pacientes por cada millón de habitantes ${ }^{20}$, siendo las causas iatrogénicas las más frecuentes. Cerca de $1 \%$ de la población general utiliza corticoides prolongadamente y dos tercios desarrollan esta patología ${ }^{4}$.

Junto al fenotipo clásico, este síndrome eleva en 4 veces el riesgo de presentar eventos cardiovasculares mayores (en especial enfermedad coronaria e insuficiencia cardiaca), en relación a la población general ${ }^{4-20}$. Al regresar el síndrome, el riesgo disminuye parcialmente ${ }^{20}$.
Por estos motivos, es fundamental evitar nuevos casos y estudiar a usuarios $\operatorname{crónicos}^{20}$. El estudio de hipercortisolismo se realiza con un test de screening y otro confirmatorio.

Existen 3 test de screening con similar rendimiento: CLU, cortisol salival nocturno y test de Nugent (TN). Si uno de ellos está alterado, se debe realizar un $\mathrm{TN}_{\mathrm{o}} \mathrm{TL}^{18}$.

Confirmado el hipercortisolismo, el estudio etiológico se efectúa midiendo $\mathrm{ACTH}^{17}$. Nosotros identificamos hipercortisolismo ACTH independiente con CLU bajo; este último debe hacer sospechar presencia de CE. Se realizó TL, pues la 
secreción ACTH neoplásica es inhibida con dosis bajas de dexametasona, a diferencia de $\mathrm{CE}$, en que no hay inhibición ${ }^{17}$.

El estudio imagenológico de glándulas suprarrenales permite detectar adenomas, neoplasias o hiperplasia que sean responsables de hipercortisolismo $^{18}$. En este reporte se descartó este origen, por lo cual, se concluyó como causa CE.

Fluticasona es un CI que es metabolizado a nivel hepático por la isoenzima CYP3A4, que da origen al derivado 17-carboxílico. Habitualmente, su actividad sistémica es mínima en dosis de 100-250 $\mu$ g día ${ }^{5}$.

Sin embargo, altas dosis de CI pueden generar efectos sistémicos. El marcador más sensible de actividad sistémica de CI es la evaluación del eje HHA a través de cortisol basal, que en estos casos es $<15 \mathrm{ug} / \mathrm{dl}^{6}$.

Por otra parte, ritonavir es un IP que también es metabolizado por la CYP3A4; determinando el descenso de la actividad del sistema enzimático citocromo P450, por unión competitiva reversible ${ }^{7}$.

Es así como, ritonavir provoca un aumento de los niveles plasmáticos de fluticasona a través de la inhibición de esta isoenzima, lo cual incrementa 200 veces los niveles plasmáticos de CI y determina hipercortisolismo ${ }^{3}$. El cual, al ser sostenido en el tiempo, suprime el eje HHA y puede dar origen a $\mathrm{SCI}^{3}$.

En consecuencia, resulta razonable considerar que tratamientos que incluyan ritonavir y fluticasona son aun más deletéreos que altas dosis de $\mathrm{CI}^{3}$. Este punto, sumado a la regresión del cuadro tras la suspensión de los fármacos, explica que el caso presentado sea secundario a esta asociación, más que, sólo a dosis elevadas de CI.

Del mismo modo, se ha reportado con mayor frecuencia esta interacción en población adulta $^{3,9,10}$. Es por ello que la OMS, desde el año 2003, alerta y recomienda no utilizar concomitantemente ritonavir y fluticasona en el tratamiento de adultos ${ }^{11}$.

En Chile no hay reportes, por lo cual, es importante alertar a los clínicos y realizar estudios que permitan conocer las comorbilidades más frecuentes en portadores de VIH y de este modo identificar a la población potencialmente susceptible a esta interacción.

Han sido reportados 35 casos como este, siendo más frecuente en hombres con edad promedio de 48 años. Además, se ha presentado SCI con múltiples CI como: fluticasona, budesonida y beclometasona. la asociación más frecuente es para fluticasona, con dosis desde 400 ug y para ritonavir desde $100 \mathrm{mg}$. El tiempo promedio de inicio del cuadro es de 8 meses, similar al caso expuesto ${ }^{3}$.

Es relevante considerar que otros reportes documentan SCI al utilizar corticoides por otras vías como: tópica, como el clobetasol ${ }^{16-19}$ intra articular $^{14-19}$ o epidural ${ }^{15-19}$; estas últimas al usar triamcinolona y ritonavir.

En cuanto a la regresión del cuadro, se ha observado en 1 a 8 meses desde la suspensión de uno de los fármacos, con un promedio de 3,5 meses; nuestro caso forma parte del tiempo promedio ${ }^{3}$. Esta recuperación es concordante con la normalización del eje HHA, expresada en el incremento del cortisol basal (Tabla 1).

Además, es importante señalar que en portadores de VIH existe desmineralización ósea por acción del virus del VIH y diferentes fármacos (corticoides, tenofovir e IP). Los IP aumentan el riesgo de fractura en menor medida que tenofovir $(12 \% \text { cada año })^{8}$; el cual, genera estimulación de osteoclastos, inhibición de osteoblastos e hipofosfemia secundaria a menor secreción tubular proximal $^{12-13}$.

De esta forma, evitar uso conjunto de CI e IP desde el inicio del tratamiento reduce el riesgo de hospitalización, mejora la adherencia al disminuir la polifarmacia y disminuye la morbimortalidad.

Existen diversas opciones terapéuticas como agonistas $\beta 2$ de acción prolongada y diferentes antirretrovirales, que a largo plazo son una medida costo efectiva, por lo cual, es necesario considerarlos en casos potencialmente susceptibles y velar por políticas que los garanticen.

Concluimos que, el SCI sigue siendo una patología poco frecuente, tiene una elevada morbimortalidad y lenta reversibilidad.

A pesar de que el caso presentado corresponda a una complicación farmacológica inusual, está bien descrita la asociación entre CI, ritonavir y SCI. Es posible prevenir nuevos casos mediante prescripción racional de corticoides, emplearlos el menor tiempo posible, controlar a usuarios crónicos con cortisol basal y evitarlos en quienes tienen alto riesgo cardiovascular.

En los casos de población VIH, además de la estrategia anterior, se debe efectuar una evaluación exhaustiva de los fármacos que serán recetados. 
Con el propósito de nunca utilizar concomitantemente CI e IP y, de existir la asociación, se debe reemplazar uno de ellos precozmente.

Agradecimientos: Dr. Gustavo Avendaño, Radiólogo Hospital Regional de Talca.

\section{Referencias}

1. Cáceres-Burton K, Álvarez-Iturriaga P. Informe nacional: Evolución VIH/SIDA, Chile 1984-2012. Departamento de Epidemiología. División de Planificación Sanitaria Ministerio de Salud de Chile. Disponible en: http://epi.minsal.cl/epi/html/bolets/reportes/VIH-SIDA/ InformePais_1984-2012_vih_sida.pdf [Consultado el 1 de abril de 15].

2. Drummond MB, Kirk GD. HIV-associated obstructive lung diseases: insights and implications for the clinician. The Lancet Respiratory Medicine 2014; 2 (7): 58392.

3. Gómez-Cerquera JM, Hernando-López E, Blanco-Ramos JR. Insuficiencia suprarrenal iatrogénica por la interacción entre ritonavir y fluticasona inhalada. Revisión de la literatura. Enfermedades Infecciosas y Microbiología Clínica 2014; 32 (10): 662-5.

4. Fardet L, Petersen I, Nazareth I. Risk of cardiovascular events in people prescribed glucocorticoids with iatrogenic Cushing's syndrome: cohort study. BMJ 2012; 345: e4928.

5. Aguirre M, Gutiérrez S, Quian J, Ramírez MJ, González V. Síndrome de Cushing secundario a interacción medicamentosa ritonavir/fluticasona. Archivos de Pediatría del Uruguay 2012; 83 (1): 21-5.

6. Eugenio A, René B. Tratamiento con Glucocorticoides. En: Eugenio A, René B, Editores, Manual de endocrinología clínica. Santiago, Chile: Editorial Mediterráneo Ltda; 2013. p. 139.

7. Melani AS. Nebulized corticosteroids in asthma and COPD. An Italian appraisal. Respiratory care 2012; 57 (7): 1161-74.

8. Roger B, Naim M, Song Z, Henning D, Pablo T. Osteoporotic fracture risk associated with cumulative exposure to tenofovir and other antiretroviral agents. AIDS 2012; 26; 825-31.
9. Foisy M, Yakiwchuk E, Chiu I, Singh A. Adrenal suppression and Cushing's syndrome secondary to an interaction between ritonavir and fluticasone: a review of the literatura. HIV Medicine 2008; 9: 389-96.

10. Mahlab-Guri K, Asher I, Gradstein S, Zung A, Radian-Sade S, Elbirt D, et al. Inhaled fluticasone causes iatrogenic cushing's syndrome in patients treated with Ritonavir. Journal of Asthma 2011; 48 (8): 860-3.

11. WHO Pharmaceuticals Newsletter 2004, No 02. Disponible en: http://apps.who.int/medicinedocs/es/d/ Js4975e/1.6.html [Consultado el 1 de abril de 15].

12. William G. Osteoporosis and Bone Health in HIV. Curr HIV/AIDS Rep 2012; 9: 218-22.

13. Adrian SR, Marshall WF, Michael JM. Tenofovir alafenamide: A novel prodrug of tenofovir for the treatment of Human Immunodeficiency Virus. Antiviral Research 2016; 125: 63e70.

14. Gregor J, Diana O, Patrick M, Markus H, Caroline FS, Alexandra C. Clinical Course and Management of Iatrogenic Cushing's Syndrome after Co-Administration of Injected-Triamcinolone and Ritonavir: a Systematic Review. J Antivir Antiretrovir 2013; 5: 180-4.

15. Matthew JG, Mark AH. Iatrogenic Cushing Syndrome After Epidural Steroid Injections for Lumbar Radiculopathy in an HIV-Infected Patient Treated With Ritonavir: A Case Report Highlighting Drug Interactions for Spine Interventionalists. American Academy of Physical Medicine and Rehabilitation 2012; 4: 234-7.

16. Sem D, Veronica F, Elisa B, Andrea S, Giovanni L. Iatrogenic Cushing's syndrome and topical steroid therapy: case series and review of the literature. Journal of Dermatological Treatment 2014; 25: 495-500.

17. Hershel R, Ty C. Cushing's syndrome: from physiological principles to diagnosis and clinical care. J Physiol 2015; 593 (3): 493-506.

18. Nieman LK, Biller BM, Findling JW, Newell-Price J, Savage MO, Stewart PM, et al. The diagnosis of Cushing's syndrome: an Endocrine Society Clinical Practice Guideline. J Clin Endocrinol Metab 2008; 93 (5): 152640.

19. Susmeeta TS, Lynnette KN, Richard AF. Cushing's syndrome: epidemiology and developments in disease management. Clinical Epidemiology 2015; 7: 281-93.

20. André L, Richard AF, Constantine AS, Lynnette KN. Cushing's syndrome. Lancet 2015; 386: 913-27. 